\title{
FERIMENTOS PENETRANTES TÓRACO-ABDOMINAIS E DE TÓRAX E ABDOME: ANÁLISE COMPARATIVA DA MORBIDADE E MORTALIDADE PÓS-OPERATÓRIAS
}

\author{
PENETRATING THORACOABDOMINAL AND ISOLATED THORACIC AND \\ ABDOMINAL WOUNDS: COMPARATIVE ANALYSIS OF POSTOPERATIVE \\ MORBIDITY AND MORTALITY
}

\author{
Jacqueline Arantes Giannini, TCBC-SP ${ }^{1}$ \\ Samir Rasslan, TCBC-SP ${ }^{2}$ \\ Leonardo Emílio da Silva, TCBC-SP ${ }^{1}$ \\ Raul Coimbra, TCBC-SP ${ }^{3}$ \\ Roberto Saad Júnior, TCBC-SP ${ }^{4}$
}

\begin{abstract}
RESUMO: Analisamos 145 doentes portadores de ferimentos penetrantes tóraco-abdominais e de tórax e abdome, operados no Serviço de Emergência da Santa Casa de São Paulo de julho de 1987 a fevereiro de 1996, sendo 72 (49,7\%) produzidos por arma branca e $73(50,3 \%)$ por projétil de arma de fogo. Foram estudados fatores relacionados à ocorrência de complicações pós-operatórias (pleuropulmonares, abdominais e sistêmicas), ao prolongamento do tempo de permanência hospitalar e à mortalidade ocorrida durante a internação. Caracterizamos os doentes quanto a sua gravidade, através da aplicação de índices objetivos de trauma, tanto fisiológico (RTS) quanto anatômicos (ISS, PATI, PTTI e PTI). Tanto nos ferimentos tóracoabdominais quanto de tórax e abdome, o tratamento de escolha foi a drenagem pleural associada à laparotomia exploradora. Os ferimentos tóraco-abdominais apresentaram maior incidência de complicações em geral, em relação aos de tórax e abdome, quando a variável controle foi o ferimento produzido por arma branca. A análise por tipo de complicação mostrou que essa diferença foi dada pelo empiema pleural. Não encontramos diferença significante entre esses ferimentos com relação às demais complicações pleuropulmonares infecciosas, abdominais e sistêmicas. Os fatores que se correlacionaram com a evolução para empiema foram: o tipo de órgão lesado (estômago, esôfago e reto), a presença de fístula digestiva, o ferimento produzido por arma branca e a presença de lesão diafragmática. O prolongamento do tempo de permanência hospitalar foi determinado pela ocorrência de complicações e não pela lesão diafragmática. Houve doze $(8,3 \%)$ mortes no estudo, sendo que a mortalidade correlacionou-se com maior média de lesões orgânicas por doente, com as lesões de rim, grandes vasos e esôfago, com a ocorrência de complicações especialmente de natureza infecciosa e com o ferimento produzido por projétil de arma de fogo. A análise dos nossos resultados permitiu concluir que os ferimentos penetrantes tóraco-abdominais apresentam maior número de lesões orgânicas por doente quando comparados aos ferimentos de tórax e abdome (sem lesão diafragmática), mas esses ferimentos não diferem quanto à mortalidade pós-operatória. Com relação à morbidade, a lesão diafragmática não foi fator determinante do prolongamento do tempo de permanência hospitalar e, na comparação dos ferimentos tóraco-abdominais e de tórax e abdome, a lesão diafragmática produzida por arma branca foi fator determinante do aparecimento de empiema pleural.
\end{abstract}

Unitermos: Trauma de tórax; Ferimentos tóraco-abdominais; Ferimentos torácico e abdominal.

\footnotetext{
1. Assistente do Serviço de Emergência. Mestre em Medicina.

2. Professor Titular e Diretor do Serviço de Emergência.

3. Professor Assistente do Serviço de Emergência.

4.Professor Titular da Disciplina de Cirurgia Torácica.
}

Recebido em 25/11/97

Aceito para publicação em 18/6/98

Trabalho realizado no Serviço de Emergência do Departamento de Cirurgia da Faculdade de Ciências Médicas da Santa Casa de São Paulo. 


\section{INTRODUÇÃO}

O aumento da incidência dos ferimentos penetrantes produzidos por instrumentos cortantes e por projéteis de arma de fogo nos centros urbanos vem sendo destacado por vários autores na literatura, ${ }^{1,2}$ sendo o tórax e o abdome as regiões do corpo mais expostas a esse tipo de ferimento.

A maioria dos traumatismos isolados de tórax pode ser tratada com sucesso com medidas relativamente simples, mesmo quando produzido por projétil de arma de fogo, ${ }^{3,4}$ sendo a toracotomia necessária em $5 \%$ a $25 \%$ dos traumatizados. ${ }^{5,6}$ Com relação às lesões extratorácicas associadas ao ferimento torácico penetrante, destacam-se as lesões abdominais por representarem significativa fonte de morbidez e mortalidade. Essas lesões comumente resultam de ferimentos transdiafragmáticos, embora possam ser produzidos diretamente por ferimento abdominal em associação e independente do ferimento torácico,

Quanto aos ferimentos diafragmáticos, muitas controvérsias existem com relação ao diagnóstico, prioridades de atendimento e conduta. Os estudos mais recentes dão ênfase aos aspectos diagnósticos da lesão diafragmática destacando sua importância devido à significante morbidez e até mortalidade tardias em função de lesões identificadas tardiamente ou não identificadas. ${ }^{7,8,9}$

Ênfase tem sido dada à análise da mortalidade dos ferimentos penetrantes mais do que à sua morbidez, o que parece justificar a pouca compreensão dos fatores de risco para o desenvolvimento de complicações pós-operatórias particularmente de natureza infecciosa desses ferimentos, ${ }^{10,11}$ apesar do número crescente de doentes que sobrevivem ao atendimento inicial, entrando na fase de risco para esse tipo de complicação.

Considerando o aumento dos ferimentos penetrantes que atingem as cavidades pleural e abdominal, optamos pelo estudo comparativo dos ferimentos penetrantes tóraco-abdominais (com lesão diafragmática) e de tórax e abdome concomitantes (sem lesão diafragmática) com o objetivo de analisar os principais fatores associados à ocorrência de complicações e mortalidade pós-operatórias em função da presença ou não da lesão do diafragma.

\section{MATERIAL E MÉTODOS}

Analisamos 145 prontuários de doentes vítimas de ferimentos penetrantes torácico e abdominal e tóraco-abdominal, operados no Serviço de Emergência do Departamento de Cirurgia da Faculdade de Ciências Médicas da Santa Casa de São Paulo no período de julho de 1987 a fevereiro de 1996.

Nesta casuística, $93(64,1 \%)$ doentes foram vítimas de ferimento penetrante tóraco-abdominal e 52 (35,9\%) de ferimentos torácico e abdominal exclusivos. Em 72 casos $(49,7 \%)$, os ferimentos foram produzidos por arma branca e, em 73 (50,3\%), por projétil de arma de fogo. A maioria dos doentes, 137 (94,5\%), era do sexo maculino. A idade variou de 15 a 64 anos, com média de idade de $29 \pm 9$ anos sendo que $93,8 \%$ pertenciam à faixa etária entre 15 e 44 anos.

Com relação ao estado fisiológico dos doentes à admissão, $125(86,2 \%)$ apresentavam-se com pressão arterial sistólica acima de 80mmHg, 99 (68,3\%) encontravam-se eupnéicos e 137 (94,5\%) estavam conscientes à admissão.

Entre os ferimentos tóraco-abdominais, a drenagem torácica foi feita em $85(91,4 \%)$ doentes, sendo em 18 (21,2\%) realizada na sala de emergência, o mesmo ocorrendo com nove $(18,8 \%)$ dos $48(92,3 \%)$ doentes drenados portadores de ferimentos torácico e abdominal. Quatro doentes vítimas de ferimento torácico e abdominal não foram drenados.

A drenagem foi o tratamento definitivo para o ferimento torácico em $74(78,5 \%)$ casos de ferimentos tóraco-abdominais e em $45(86,5 \%)$ de ferimentos de tórax e abdome. A toracotomia foi realizada em 12 doentes, sendo dez (10,8\%) do primeiro grupo e dois $(3,9 \%)$ do segundo; a esternotomia foi feita em apenas dois doentes, um de cada grupo.

A via de acesso preferencial para a cavidade abdominal foi a laparotomia mediana realizada em 142 (97.9\%) doentes, sendo três doentes tratados por videolaparoscopia.

A drenagem torácica associada à laparotomia exploradora foi a conduta adotada em $91,4 \%$ dos doentes com ferimentos tóraco-abdominais e em $88,5 \%$ dos torácicos e abdominais. A partir do achado operatório, os doentes foram divididos como sendo portadores de ferimento penetrante tóraco-abdominal (TA), fazendo parte desse grupo 93 doentes, e de tórax e abdome (T+A), 52 doentes, conforme a presença ou não de lesão diafragmática. No estudo desses ferimentos consideramos também o mecanismo de trauma, subdividindo-os em ferimentos produzidos por arma branca (FAB) e por projétil de arma de fogo (PAF), resultando assim em quatro grupos para estudo: ferimentos tóraco-abdominais produzidos por arma branca (TA/FAB), 49 doentes; ferimentos tóraco-abdominais por projétil de arma de fogo (TA/PAF), 44 doentes; ferimentos de tó-rax e abdome produzidos por arma branca ( $\mathrm{T}+\mathrm{A} / \mathrm{FAB})$, 23 doentes, e ferimentos de tórax e abdome por projétil de arma de fogo, (T+A/PAF), 29 doentes.

As complicações pós-operatórias consideradas foram divididas em pleuropulmonares infecciosas (pneumonia com e sem derrame pleural e empiema pleural), pleuropulmonares não infecciosas (hemotórax, pneumotórax, derrame não pneumônico), abdominais e sistêmicas. As principais complicações abdominais analisadas foram: infecção da ferida cirúrgica, fístula digestiva, abscesso intraperitoneal e peritonite. As complicações sistêmicas analisadas foram: insuficiência respiratória aguda, insuficiência renal aguda e sepse.

A gravidade dos doentes foi avaliada objetivamente através da utilização do Revised Trauma Score (RTS), ${ }^{1}$ do Penetrating Abdominal Trauma Index (PATI), ${ }^{10}$ Penetrating Thoracic Trauma Index (PTTI), ${ }^{13}$ e do Injury Severity Score (ISS), ${ }^{14}$ calculados a partir da avaliação das lesões anatômicas encontradas. 
FERIMENTOS PENETRANTES TÓRACO-ABDOMINAIS E DE TÓRAX E ABDOME: ANÁLISE COMPARATIVA DA MORBIDADE E MORTALIDADE PÓS-OPERATÓRIAS

A mortalidade a partir do pós-operatório imediato foi analisada em função do local do trauma (TA ou T+A), do mecanismo (FAB ou PAF) e dos índices de trauma. A probabilidade de sobrevida dos doentes foi avaliada pelo método do TRISS, que combina parâmetros fisiológico (RTS) e anatômico (ISS) com o mecanismo de trauma e idade dos doentes.(

A análise estatística foi feita com a aplicação do Teste t de Student, controlado pelo Teste de Levine, para a comparação entre as proporções das variáveis dicotômicas e ordinais. Para a análise das relações entre as variáveis foi utilizado o Teste de Qui-Quadrado, e para as relações com significância estatística foi aplicada a Correlação de Pearson. O nível de significância adotado foi $5 \%(\mathrm{p}<0,05)$.

\section{RESULTADOS}

Os ferimentos tóraco-abdominais apresentaram maior média $(\mathrm{p}<0,001)$ de lesões orgânicas por doente $(2,85)$ quando comparados aos torácico e abdominal $(1,77)$, independente do mecanismo de trauma considerado. As lesões esplênica e gástrica predominaram nos ferimentos com lesão diafragmática, enquanto as lesões intestinais, especialmente de delgado, foram mais freqüentes nos ferimentos de tórax e abdome. Das lesões intratorácicas, a lesão pulmonar foi a mais comum em todos os grupos considerados e também não houve diferença $(p>0,05)$ entre os grupos quanto às lesões extrapulmonares (coração, artéria mamária interna e vasos intercostais).

Com relação aos índices de trauma, não houve diferença significante entre as médias do RTS por local e mecanismo de trauma.

A média do ISS dos 145 doentes foi 24,1. A média do ISS dos ferimentos tóraco-abdominais $(21,3)$ foi maior $(\mathrm{p}<0,001)$ do que a média dos ferimentos de tórax e abdome $(16,7)$ quando a variável controle foi o ferimento produzido por arma branca. A comparação das médias do ISS entre os demais grupos não mostrou diferença $(p>0,05)$.

A média do PATI foi 13,5 e do PTI de 18,4 . Não houve diferença significante na comparação das médias desses índices entre os grupos, independente do local e do mecanismo de trauma. Com relação ao componente torácico, a média do PTTI foi 4,9 e também não houve diferença entre os grupos. Setenta e seis doentes $(52,4 \%)$ evoluíram com complicações, sendo $52(55,9 \%)$ vítimas de ferimentos tóraco-abdominais e $24(46,2 \%)$ vítimas de ferimentos de tórax e abdome concomitantes $(\mathrm{p}>0,05)$.

Os ferimentos tóraco-abdominais apresentaram maior incidência $(p=0,040)$ de complicações que os ferimentos de tórax e abdome apenas quando a variável controle foi o ferimento produzido por arma branca. Essa diferença foi dada pelo empiema pleural, não havendo diferença com relação às demais complicações (Tabelas 1 e 2).

Os fatores que se correlacionaram com a ocorrência de complicações em geral foram: o número de lesões orgânicas
Tabela 1

Complicações nos ferimentos tóraco-abdominais e de tórax e abdome

\begin{tabular}{l|c|l|c}
\hline & TA & T+A & $p$ \\
\hline FAB & $51,0 \%$ & $26,1 \%$ & $0,040 *$ \\
PAF & $61,4 \%$ & $62,1 \%$ & 0,952 \\
Total & $55,9 \%$ & $46,2 \%$ & 0,259 \\
\hline
\end{tabular}

* Significância com nível descritivo inferior a $5 \%$

Tabela 2

Complicações pleuropulmonares

\begin{tabular}{lcccc}
\hline Complicações & TA $(\mathrm{N}=93)$ & $\mathrm{T}+\mathrm{A}(\mathrm{N}=52)$ & $\mathrm{p}$ \\
\hline Pneumonia & $16(17,2 \%)$ & $10(19,2 \%)$ & 0,762 \\
Pneumonia com & $3(3,2 \%)$ & $1(1,9 \%)$ & 0,649 \\
$\quad$ derrame pleural & $10(10,8 \%)$ & $1(1,9 \%)$ & $0,020 *$ \\
Empiema pleural & $3(3,2 \%)$ & $0(0,0 \%)$ & 0,083 \\
Atelectasia & $6(6,5 \%)$ & $4(7,7 \%)$ & 0,779 \\
Pneumotórax & $14(15,1 \%)$ & $3(5,8 \%)$ & 0,063 \\
Hemotórax & $1(1,1 \%)$ & $0(0,0 \%)$ & 0,457 \\
Derrame pleural & $\quad$ & & \\
\multicolumn{1}{c}{ não pneumônico } &
\end{tabular}

* Significância com nível descritivo inferior a 5\%

por doente, o tipo de órgão lesado, o mecanismo de trauma e alguns índices de trauma (RTS, ISS, PATI e PTI), conforme mostra a tabela 3 .

Tabela 3

Índices de trauma e complicações em geral

\begin{tabular}{l|c|c|c}
\hline Índices & $\begin{array}{l}\text { Sem } \\
\text { complicação }\end{array}$ & $\begin{array}{l}\text { Com } \\
\text { complicação }\end{array}$ & $\mathrm{p}$ \\
\hline RTS & 7,7 & 7,2 & $0,015^{*}$ \\
PATI & 10,0 & 16,8 & $0,001^{*}$ \\
PTTI & 4,7 & 5,0 & 0,519 \\
PTI & 14,7 & 21,8 & $0,001^{*}$ \\
ISS & 21,5 & 26,4 & $0,001^{*}$ \\
\hline
\end{tabular}

* Significância com nível descritivo inferior a 5\%

A evolução para empiema pleural foi determinada por lesões orgânicas específicas (estômago, esôfago e reto), pela presença de fístula digestiva, pelo ferimento produzido por arma branca e pela presença de lesão diafragmática.

Não houve diferença entre os grupos quanto à ocorrência de complicações abdominais (Tabela 4), porém, observamos correlação positiva $(\mathrm{p}<0,001)$ entre essas complicações e a ocorrência de complicações pleuropulmonares infecciosas apenas nos doentes com lesão diafragmática.

A média do tempo de permanência hospitalar para os 145 doentes foi de 9,3 dias sem diferença quando considerados o local e o mecanismo de trauma, sendo o seu prolongamento determinado pela ocorrência de complicações e não pela lesão diafragmática.

A mortalidade encontrada foi compatível com a esperada em cada grupo através do cálculo do TRISS (Tabela 5). Houve 
Tabela 4

Complicações abdominais

\begin{tabular}{lcccc}
\hline Complicações abdominais & TA (N=93) & T+A (N=52) & $p$ \\
\hline Infecção da ferida cirúrgica & $12(12,9 \%)$ & $5(9,6 \%)$ & 0,558 \\
Abscesso intraperitoneal & $7(7,5 \%)$ & $3(5,8 \%)$ & 0,691 \\
Fístula digestiva & $4(4,3 \%)$ & $2(3,8 \%)$ & 0,896 \\
Peritonite & $1(1,1 \%)$ & $2(3,8 \%)$ & 0,343 \\
Evisceração & $3(3,2 \%)$ & $0(0,0 \%)$ & 0,083 \\
Hemorragia & $2(2,2 \%)$ & $0(0,0 \%)$ & 0,158 \\
HDA & $1(1,1 \%)$ & $1(1,9 \%)$ & 0,677 \\
Obstrução & $1(1,1 \%)$ & $0(0,0 \%)$ & 0,457 \\
Pancreatite & $1(1,1 \%)$ & $0(0,0 \%)$ & 0,457 \\
\hline
\end{tabular}

* Significância com nível descritivo inferior a 5\%

Tabela 5

Distribuição do TRISS por grupo e mortalidade

\begin{tabular}{l|c|c|c}
\hline Grupo & Média do TRISS & $\begin{array}{c}\text { Mortalidade } \\
\text { esperada }\end{array}$ & $\begin{array}{c}\text { Mortalidade } \\
\text { observada }\end{array}$ \\
\hline TA / FAB & 0,97 & $3,0 \%$ & $4,1 \%$ \\
TA / PAF & 0,88 & $12,0 \%$ & $13,6 \%$ \\
T+A / FAB & 0,99 & $1,0 \%$ & $0,0 \%$ \\
T+A / PAF & 0,80 & $20,0 \%$ & $13,8 \%$ \\
TA & 0,88 & $12,0 \%$ & $8,6 \%$ \\
T+A & 0,93 & $7,0 \%$ & $7,7 \%$ \\
\hline
\end{tabular}

Tabela 6

Causas de morte

\begin{tabular}{l|c|c|c|c}
\hline \multirow{2}{*}{ Causa de morte } & \multicolumn{4}{c}{ Número de mortes } \\
\cline { 2 - 5 } & TA & T+A & Total & Percentual \\
\hline Sepsis & 3 & 3 & 6 & $50,0 \%$ \\
Insuficiência respiratória & & & & \\
$\quad$ aguda & 2 & 0 & 2 & $16,7 \%$ \\
Hipovolemia & 2 & 0 & 2 & $16,7 \%$ \\
Insuficiência renal aguda & 1 & 0 & 1 & $8,3 \%$ \\
Neurológica & 0 & 1 & 1 & $8,3 \%$ \\
\hline Total & 8 & 4 & 12 & $100,0 \%$ \\
\hline
\end{tabular}

doze mortes nesse estudo, sem diferença significante entre os ferimentos tóraco-abdominais e de tórax e abdome, com maior mortalidade entre as vítimas de ferimentos produzidos por projéteis de arma de fogo.

A sepse respondeu por 50,0\% das mortes, seguida da insuficiência respiratória aguda $(16,7 \%)$, da hipovolemia $(16,7 \%)$, insuficiência renal aguda $(8,3 \%)$ e das causas neurológicas $(8,3 \%)$, conforme mostra a tabela 6 .

A mortalidade correlacionou-se com maior média de lesões orgânicas por doente, lesões de rins, grandes vasos, esôfago, duodeno e fígado; com a ocorrência de complicações, especialmente de natureza infecciosa (empiema pleural, pneumonia e peritonite), com a gravidade anatômica avaliada pelo ISS, PATI, PTI e com o ferimento produzido por projétil de arma de fogo.

\section{DISCUSSÃO}

Com a violência dos centros urbanos, as lesões intencionais, tanto produzidas por arma branca quanto por projétil de arma de fogo, passaram a ser a principal causa de morte na população jovem. Nesse contexto, os ferimentos diafragmáticos têm se tornado comuns, pois podem estar associados a lesões torácicas e/ou abdominais. ${ }^{15,16}$ A maioria dos trabalhos referentes à violação concomitante das cavidades torácica e abdominal discute problemas relativos ao diagnóstico da lesão diafragmática sem analisar propriamente os fatores envolvidos na morbidez e mortalidade precoces desse tipo de lesão. Estudamos esses fatores através de um estudo comparativo entre os ferimentos tóraco-abdominais e de tórax e abdome (sem lesão diafragmática).

A drenagem torácica associada à laparotomia exploradora foi a conduta adotada na maioria dos doentes independente de tratar-se de ferimento tóraco-abdominal ou de tórax e abdome. Nossa incidência de cirurgia no tórax de 9,7\% correlacionou-se com a gravidade anatômica dos doentes avaliada pelo ISS, PTI e PTTI e, portanto, não resultou exclusivamente do componente torácico. Observamos que em dois casos o sangramento era de origem abdominal, em dois, a lesão pulmonar encontrada à cirurgia não justificava o procedimento e em um doente a toracotomia foi realizada para controle de empiema pleural.

Com relação às lesões abdominais encontradas, os dois tipos de ferimentos cursaram com lesões orgânicas de risco para a instalação de complicações sépticas. A lesão isolada do diafragma ocorreu em 7,5\% dos casos, sendo essa incidência compatível com a relatada pela maioria dos autores consultados.

Para identificar os doentes com maior risco de desenvolver complicações pós-operatórias e com menor probabilidade de sobrevida utilizamos os índices de trauma. Do ponto de vista fisiológico (RTS) não houve diferença quanto à gravidade dos doentes à admissão entre as vítimas de ferimentos tóraco-abdominais e de tórax e abdome.

A gravidade anatômica foi avaliada pelo ISS, que expressa o efeito cumulativo das lesões mais graves das três regiões do corpo mais atingidas e, com relação a esse índice, só houve diferença entre os ferimentos tóraco-abdominais e de tórax e abdome quando a variável controle foi o ferimento produzido por arma branca. Nesse caso, maior ISS (maior gravidade) foi encontrada entre as vítimas de ferimentos tóraco-abdominais. No cálculo do ISS, a lesão do diafragma é avaliada como parte do conteúdo abdominal e pélvico e, como nos ferimentos por arma branca há maior chance de penetração na cavidade peritoneal sem lesão visceral ou com lesões menos graves, ${ }^{20,21,22}$ a presença da lesão diafragmática pode justificar o mais alto valor do ISS para os ferimentos tóracoabdominais para esse mecanismo de trauma.

Para analisarmos a extensão total do trauma utilizamos ainda o PTI (PATI + PTTI) cuja comparação das médias dos 
grupos mostrou que a gravidade anatômica dos ferimentos foi dada pelo mecanismo de trauma (PAF) e não pela lesão diafragmática.

A gravidade dos doentes vem sendo associada à presença de complicações pleuropulmonares infecciosas, ${ }^{23,24} \mathrm{e}$ em nosso estudo encontramos correlação positiva entre os mais altos valores de ISS e PTI e à ocorrência de pneumonia, que foi a complicação pleuropulmonar infecciosa mais comum, embora sua incidência real seja difícil de determinar em função da sensibilidade e especificidade dos critérios e métodos diagnósticos. Adotamos critérios clínicos e radiológicos ${ }^{25,26}$ para todos os doentes e não encontramos diferença entre os ferimentos tóraco-abdominais e de tórax e abdome quanto à ocorrência dessa complicação.

Embora alguns autores destaquem a maior gravidade associada aos ferimentos tóraco-abdominais em relação aos ferimentos isolados de tórax ou de abdome,,$^{20,19,27}$ encontramos maior incidência de complicações em geral nos ferimentos com lesão diafragmática apenas quando a arma branca foi o agente agressor, e esse resultado é compatível com o maior ISS encontrado nesse grupo. A arma branca produz ferimentos mais extensos no diafragma e isso pode justificar a maior incidência de complicações nos ferimentos tóraco-abdominais quando esse foi o agente agressor, uma vez que a diferença entre esses ferimentos e os de tórax e abdome foi dada quase exclusivamente pelo empiema pleural.

Considerando-se que os dois tipos de ferimentos cursaram com lesões abdominais com alto poder de contaminação, o tipo de lesão produzida no diafragma pela arma branca provavelmente facilitou a contaminação do espaço pleural. Bernini (1991) encontrou correlação positiva entre a presença de lesões diafragmáticas maiores que $2 \mathrm{~cm}$ e a ocorrência de pneumonia e empiema pleural.

O empiema pleural foi a única complicação pleuropulmonar infecciosa cuja incidência variou entre os grupos de forma significante. As lesões de cólon e, mais recentemente, de estômago vêm sendo destacadas na literatura como principais fatores de risco para o aparecimento dessa complicação. Bernini (199.1) destacou ainda a associação de fístula digestiva e empiema pleural, particularmente a fístula gástrica. Em nosso estudo, não houve diferença entre os ferimentos tóracoabdominais e de tórax e abdome com relação à ocorrência de complicações abdominais, mas só encontramos correlação positiva entre a fístula digestiva e empiema quando na presença de lesão diafragmática. Os ferimentos tóraco-abdominais e de tórax e abdome produzidos por arma branca não diferiram quanto à frequiência de lesão de estômago e de fístula digestiva, o que reforça a importância do mecanismo de trauma (ferimento por arma branca) e não só a lesão diafragmática na evolução para empiema pleural. Quando a variável controle foi o ferimento por projétil de arma de fogo, não encontramos diferenças entre os grupos com relação a essa complicação.
Com relação à ocorrência de complicações abdominais não encontramos diferenças entre os grupos, sendo a infecção da ferida cirúrgica a complicação mais comum atingindo os doentes mais graves (mais altos valores de ISS, PATI e PTI) e normalmente acompanhou outras complicações abdominais (abscesso intraperitoneal, peritonite e fístula digestiva).

Em nosso estudo, e em concordância com a literatura, a ocorrência dessas complicações envolveu especialmente as lesões de estômago, cólon, esôfago, fígado, rim e duodeno, ${ }^{28,29}$ que foram consideradas para o cálculo dos índices de trauma.

A morbidez dos ferimentos penetrantes estudados foi avaliada também em função do tempo de permanência hospitalar, sendo o fator determinante do seu prolongamento a ocorrência de complicações e não a presença de lesão diafragmática.

As complicações infecciosas responderam pela maioria das mortes ocorridas nesse estudo, resultado que é concordante com o encontrado na literatura consultada. ${ }^{30,19}$

Moore et al (1981) destacam a multiplicidade de lesões orgânicas como a principal determinante da mortalidade, embora o tipo de lesão também seja importante, especialmente as de fígado, duodeno, baço e grandes vasos. No presente estudo, com exceção do baço, as demais lesões correlacionaram-se positivamente com a mortalidade e a média de lesões por doente também. Dos sete doentes com lesão isolada do diafragma, nenhum morreu, reforçando a importância das lesões associadas.

A mortalidade entre os grupos variou com a gravidade anatômica que determinou mais altos valores de ISS, PATI e PTI, sendo compatível com a esperada pela aplicação da metodologia do TRISS. Não foi observada diferença entre as médias do RTS dos mortos e sobreviventes, sugerindo que, nesse estudo, o estado físiológico dos doentes à admissão não influenciou a mortalidade pós-operatória, o que foi interpretado como decorrente do critério de seleção adotado.

Alguns autores consideram que a mortalidade está diretamente relacionada ao mecanismo de trauma, especialmente ao ferimento por projétil de arma de fogo. ${ }^{13,19,27,2}$

Encontramos maior mortalidade nos ferimentos em que esse foi o agente agressor, independente da presença ou não de lesão diafragmática.

Assim, concluímos que os ferimentos penetrantes tóracoabdominais apresentam maior número de lesões orgânicas por doente quando comparados aos ferimentos de tórax e abdome (sem lesão diafragmática), mas esses ferimentos não diferem quanto à mortalidade pós-operatória.

Com relação à morbidez, na comparação dos ferimentos tóraco-abdominais e de tórax e abdome, a lesão diafragmática produzida por arma branca foi fator determinante do aparecimento de empiema pleural. No entanto, a lesão diafragmática, por si, não foi fator determinante do prolongamento do tempo de permanência hospitalar. 


\begin{abstract}
We retrospectively reviewed the medical records of 145 patients with diagnosis of thoracoabdominal injuries and thoracic and abdominal injuries, who underwent surgery in the Emergency Service of Santa Casa de São Paulo, from July 1987 to February 1996. Stab wounds occurred in 72 patients and gunshot wounds in the remaining 73 . We focused the analysis on factors related to postoperative complications, length of stay and mortality. Patients were classified using trauma severity score, both physiological (RTS) and anatomical (ISS, PATI, PTTI and PTI). Thoracotomy was correlated with complications. Thoracoabdominal injuries, in general, showed greater incidence of complications when the control variable was stab wounds. The analysis by complication types proved that such difference was caused by pleural empyema. Factors correlating to the occurrence of pleural empyema were: type of injuried (stomach, esophagus and rectum), presence of digestive fistula, stab wounds, and diaphragmatic wound. Length of stay increased due to the occurrence of complications, and not because of the presence of a diaphragmatic wound. Death rates were closely related with increased number of injuries, renal wounds, great vessels and esophageal injuries, infections and gunshot wounds. We concluded that a greater number of organic damages was observed in those patients sustaining penetrating thoraco-abdominal wounds, despite the same postoperative mortality, when compared with those compromised with thoracic and abdominal wounds (without diaphagmatic injury). Regarding morbidity, the increased incidence of pleural empyema in the thoracoabdominal injuries was related with diapragmatic injury by stab wounds.
\end{abstract}

Key Words: Thoracic trauma; Thoraco-abdominal injuries; Thoracic and abdominal injuries.

\title{
REFERÊNCIAS
}

1. Ordog JG, Sheppard FG, Wasserberger SJ, et al - Infection in minor gunshot wounds. J Trauma1993; 34:358-65.

2. Elliott CD; Rodriguez A - Cost effectiveness in trauma care. Surg Clin North Am1996; 76:47-63.

3. Smith SR, Fry RW, Morabito JD, et al - Preliminary report on videothoracoscopy in the evaluation and treatment of thoracic injury. Am J Surg 1993; 166:690-4.

4. Saad Jr. R, Aguiar Neto JR, Dorgan Neto V, et al - Trauma de tórax: análise de 114 doentes. Classificação, indicação operatória, mortalidade. J Pneumol 1995; 21:79-82.

5. Mandal KA, Oparah SS - Unusually low mortality of penetrating wounds of chest. J Thorac Cardiovasc Surg 1989; 97:119-25.

6. Maattox KL, Wall Jr. MJ, Pickard LR - Thoracic trauma: general consideration and indication for thoracotomy. In: FelicianoDV,

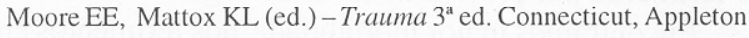
\& Lange, 1996. p.345-54.

7. Ochsner GM, Rozycki SG, Lucente F, et al - Prospective evaluation of thoracoscopy for diagnosing diafragmatic injury in thoracoabdominal trauma: a preliminary report. J Trauma 1993; 34:704-9.

8. Saad Jr. R, Rasslan S - A videocirurgia no trauma de tórax. Rev Col Bras Cir 1993; 20:III

9. Solda SC, Rodriguez FCM, Martins L, Rasslan S - Lesão diafragmática isolada por ferimento penetrante tratada por videolaparoscopia. Re. Col Bras Cir1994; 31:213-8.

10. Moore EE, Dunn LE, Moore BJ, et al - Penetrating abdominal trauma Index. J Trauma1981; 21:443-5.

11. Nichols LR, Smith WJ, Klein BD, et al - Risk of infection after penatrating abdominal trauma. N Engl J Med1984; 311:1065-70.

12. Champion RH, Sacoo JW, Copee SW, et al - A revision of the trauma score. J Trauma1989; 29: 623-9
13. Ivatury RR, Nallathambi MN, Stahl WM, et al -Penetrating cardiac trauma. Quantifying the severity of anatomic and phisiologic injury. Ann Surg 1987; 205:61-6.

14. Baker SP, O'Neill B, Haddon W - The injury severity score: a method for describing patients with multiple injuries and evaluating emergency care. J Trauma1974; 14:187-96.

15. Wiencek GR, Wilson FR, Steiger Z - Acute injuries of the diafragm. J Thorac Cardiovasc Surg 1986; 92:989-93.

16. Demetriades D, Kakoyiannis S, Parekh D, et al - Penetrating injuries of the diaphragm. Br J Surg 1988; 75:824-6.

17. Bernini OC - Ferimentos toracoabdominais: estudo de fatores determinantes de complicações pleuropulmonares. São Paulo, 1991. (Tese de Doutorado - Faculdade de Medicina da Universidade de São Paulo).

18. Durham MR, Olson S, Weigelt AJ - Penetrating injuries to the stomach. Surg Gynecol Obstet 1991;172:298-302.

19. Coimbra R, Pinto CM, Aguiar RJ, et al - Factors related to the occurrence of postoperative complications following penetrating gastric injuries. Injury 1995a; 26:463-6..

20. Moore BJ, Moore EE, Thompson SJ - Abdominal injuries associated with penetrating trauma in the lower chest. Am J Surg1980a; 140:724-30,

21. Moore EE, Moore JB, Thompson SJ, et al - Mandatory laparotomy for gunshot wounds penetrating the abdomen. Am J Surg 1980b; 140:847-51.

22. Fabian \& Croce - Abdominal trauma including indications for celiotomy. In: Feliciano DV, Moore EE, Mattox KL (ed.) - Trauma. $3^{\text {a }}$ ed. Connecticut, Appleton \& Lange, 1996. p.441-60.

23. Rodriguez LJ, Gibbons JK, Bitzer G, et al - Pneumonia: incidence, risk factors, and outcome in injuried patients. J Trauma 1991;31: 907-14. 
24. Hoyt BD, Simons RK, Winchell RJ, et al - A risk analysis of pulmonary complications following major trauma. J Trauma1993; 35:524-31.

25. Andrews CP, Coalson JJ, Smith JD - Diagnosis of nosocomial bacterial pneumonia in acute, difuse lung injury. Chest 1981; 80: 254.

26. Lode H - Initial therapy in pneumonia. Am J Med1986; 80:70-4,

27. Coimbra R, Pinto CM, Rakuk A, et al - Penetrating cardiac wounds: predictive value of trauma index and the necessity of termnology standardization. Am Surg 1995b; 61: 448-52,

28. Croce AM, Fabian CT, Stewart MR, et al - Correlation of abdominal trauma index and injury severity score with abdominal septic complications in penetrating and blunt trauma. J Trauma1992; 32:380-6.

29. Nassoura EZ, Ivatury RR, Simon JR, et al - A prospective reappraisal of primary repair of penetrating duodenal injuries. Am Surg 1993; 60: $37-9$
30. Davis WJ, Hoyt BD, McArdle SM, et al - The significance of critical care errors in causing preventable death in trauma patients in a trauma system. J. Trauma 1991;31:813-9.

\section{ENDEREÇO PARA CORRESPONDÊNCIA}

Dr. Roberto Saad Júnior

Rua dos Ingleses, 524 - $5^{\circ}$ andar

01329-000 - São Paulo - SP

\title{
XXII CONGRESSO BRASIIEIRO DE CIRURGIA
}

\author{
4 A 8 DE JULFO DE 1999 \\ RIOCENTRO \\ RIO DE JANEIRO
}

